\title{
Avaliação do módulo de elasticidade em concreto com metacaulim de alta reatividade, proveniente de rejeito industrial
}

\section{Assessment of the modulus of elasticity in concrete with high reactivity metakaolin from industry}

Paulo Sérgio Lima Souza ${ }^{1}$, Denise C. C. Dal Molin², Marcelo de Souza Picanço ${ }^{2}$, Alcebíades Negrão Macêdo ${ }^{2,}$ Adriano Luiz Roma Vasconcelos², José Victor Brasil de Souza²

\footnotetext{
${ }^{1}$ Núcleo Orientado para a Inovação da Edificação - Av. Osvaldo Aranha 99, $3^{\circ}$ andar - NORIE/UFRGS CEP: 90035 190 (51) 3308-3518, Porto Alegre, RS.

e-mail: norie@ufrgs.br

${ }^{2}$ Rua Augusto Corrêa n ${ }^{\circ}$ 01, CEP 660075-110, Belém PA

e-mail: paseliso@ufpa.br; damolin@vortex.ufrgs.br; marcelosp@ufpa.br; anmacedo@ufpa.br; adrianoroma@ufpa.br;josevictor002@hotmail.com
}

\begin{abstract}
RESUMO
As adições minerais são empregadas no concreto com o objetivo de melhorar algumas de suas propriedades mecânicas, bem como a sua durabilidade. Atualmente, tem-se cada vez mais adotado o uso de pozolanas de alta reatividade como ferramenta para obtenção do concreto de alta resistência (CAR). O metacaulim de alta reatividade (MCAR) é oriundo do processo de calcinação em temperaturas entre $400^{\circ} \mathrm{C}$ e $950^{\circ} \mathrm{C}$ e posterior processo de moagem de argilas com altos teores de caulinita, como a argila caulinítica e o caulim. O objetivo principal deste trabalho foi avaliar o uso do (MCAR) no concreto, visando verificar a sua influência no módulo de elasticidade do concreto. Para a obtenção desta adição mineral adotou-se como matéria-prima o rejeito proveniente da indústria do papel, que após um processo de calcinação e moagem, transforma-se no MCAR. Para a realização deste trabalho variou-se os teores de substituições de MCAR, as relações água/cimento e as idades de rompimento dos corpos-de-prova. A metodologia utilizada foi baseada em ensaios laboratoriais e no uso de ferramentas estatísticas para validação dos resultados. O estudo indicou que o efeito isolado das variáveis controladas e algumas interações entre essas variáveis influenciam nesta propriedade. Dentre esses efeitos isolados, constatou-se que as variações das porcentagens de MCAR de 0 a $20 \%$ resultam em acréscimos de até $9 \%$ no valor obtido no ensaio de módulo de elasticidade do concreto.
\end{abstract}

Palavras-chave: adição mineral, metacaulim de alta reatividade, módulo de elasticidade.

\section{ABSTRACT}

The mineral additives are employed in the concrete in order to improve some of its mechanical properties as well as its durability Currently, there has been increasingly adopted the use of highly reactive pozzolans as a tool for obtaining high-strength concrete (HSC). The high reactivity metakaolin (HRM) comes from the calcination process at temperatures between $400^{\circ} \mathrm{C}$ and $950^{\circ} \mathrm{C}$ and subsequent grinding process clays kaolinite with high yields, as the kaolinitic clay and kaolin. This paper aims at assessing the use of HRMK within the concrete and verifying its influence within the modulus of concrete elasticity. To obtain this mineral addition a paper industry tailing was adopted as raw material, which was then calcinated and processed in a mill to be transformed into HRMK. To accomplish this work, the content of the HRMK replacements has been varied, as well as the water/concrete ratio and the ages of disruption of the body-test. The methodology used has been based on laboratorial assays and statistical tools in order to validate the outcomes. The study concludes that the isolated effect of the controlled variables and some interactions between these variables influenced the concrete's elasticity. 
Keywords: mineral, high reactivity metakaolin, elasticity modulus.

\section{INTRODUÇÃO}

O conhecimento do módulo de elasticidade é de grande importância para a execução do projeto, pois o seu valor auxilia na previsão de deformações, no conhecimento das tensões entre o concreto e o aço das estruturas de concreto armado e protendido, além de contribuir para o cálculo das tensões resultantes de retração e de recalque. Contudo, este conhecimento ainda se encontra no estágio inicial em se tratando do comportamento desta propriedade com o uso de pozolanas de alta reatividade, principalmente quando se focaliza o uso do metacaulim de alta reatividade (MCAR).

O Metacaulim é uma adição mineral aluminossilicosa derivada normalmente da calcinação, entre $600^{\circ} \mathrm{C}$ e $900^{\circ} \mathrm{C}$, de alguns tipos de argilas, como as argilas cauliníticas e os caulins. Segundo [1], alguns pesquisadores tendem a distinguir entre o metacaulim e o metacaulim de alta reatividade (MCAR), decorrente principalmente da maior finura do segundo. Trabalhos realizados por [2,3,4] relatam benefícios tecnológicos decorrentes do emprego desta pozolana na produção do concreto.

O MCAR, além de proporcionar vantagens técnicas, ainda proporciona a possibilidade de ser obtido através do tratamento de certos tipos de resíduos, como o proveniente da indústria de papel, constituído basicamente de um caulim beneficiado de extrema alvura, finura e pureza (alto teor de caulinita Al2O3.2SiO2.2H2O), sendo transformado em uma pozolana aluminossilicosa através do processo de calcinação (de 700 à $800^{\circ} \mathrm{C}$ ) e de moagem.

Como exemplo desta situação, apresenta-se o resíduo de uma indústria localizada a noroeste do estado do Pará, que atualmente realiza o depósito deste rejeito em lagoas preparadas para esta finalidade. Contudo, este processo de armazenamento tem-se transformado em um problema ambiental devido à necessidade de grandes áreas de desmatamento. Trabalhos realizados por [1,5,6] em misturas de concreto, obtiveram melhorias em algumas propriedades, com emprego do MCAR oriundo deste rejeito.

Desta forma, o objetivo principal deste trabalho foi verificar as variações existentes no módulo de elasticidade do concreto, decorrentes da inclusão do MCAR oriundo do rejeito da indústria do papel.

\section{MATERIAIS E MÉTODOS}

\subsection{Caracterização dos Materiais}

Adotou-se areia lavada de origem quartzosa extraída do rio Jacuí e brita de origem basáltica, cujas características, estão colocadas na tabela 1. Adotou-se o cimento Portland de alta resistência inicial (CPV-ARI). O MCAR foi obtido através de calcinação à $750{ }^{\circ} \mathrm{C}$ e posterior moagem. Na figura 1 tem-se a difração de raiosx do material após a calcinação, onde se constata a baixa cristalinidade de sua estrutura. As principais características do cimento e do MCAR estão colocados na tabela 2.

Tabela 1: Valores obtidos nas características físicas dos agregados empregados

\begin{tabular}{l|l|l}
\hline DESCRIÇÃo & AREIA & BRITA \\
\hline $\begin{aligned} \text { Massa específica - NBR 9776 (ABNT, 1986) } \\
\text { - NBR 9937 (ABNT, 1987) }\end{aligned}$ & $2,62 \mathrm{~kg} / \mathrm{dm} 3$ & \\
\hline Massa unitária - NBR 7251 (ABNT, 1982) & $1,51 \mathrm{~kg} / \mathrm{dm} 3$ & $1,44 \mathrm{~kg} / \mathrm{dm} 3$ \\
\hline Modulo de finura - NBR 7217 (ABNT, 1987) & 2,43 & 6,85 \\
\hline Diâmetro máximo - NBR 7217 (ABNT, 1987) & $2,4 \mathrm{~mm}$ & $19 \mathrm{~mm}$ \\
\hline
\end{tabular}


Tabela 2: Principais características do cimento e do MCAR utilizado

\begin{tabular}{|c|c|c|c|c|c|}
\hline QUÍMICAS & CIMENTO & MCAR & FÍSICAS E MECÂNICAS & CIMENTO & MCAR \\
\hline $\mathrm{SiO}_{2}(\%)$ & 19,51 & 42,19 & Resíduo na peneira 75 m (\%) & - & 10,59 \\
\hline $\mathrm{AL}_{2} \mathrm{O}_{3}(\%)$ & 4,17 & 39,24 & Área específica $\left(\mathrm{m}^{2} / \mathrm{kg}\right)$ & 4,570 & 18,770 \\
\hline $\mathrm{Fe}_{2} \mathrm{O}_{3}(\%)$ & 2,85 & 1,88 & Diâmetro médio $(\mu \mathrm{m})$ & 9,16 & 4,65 \\
\hline$\underline{\mathrm{P}_{2} \mathrm{O}_{5}(\%)}$ & - & 0,21 & \multirow{2}{*}{$\begin{array}{l}\text { Resistência à compressão do } \\
\text { cimento }-1^{\text {o dia }} \\
\end{array}$} & \multirow[b]{2}{*}{19,00} & \multirow[t]{2}{*}{ 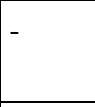 } \\
\hline $\mathrm{MgO}(\%)$ & 1,32 & 0,20 & & & \\
\hline $\mathrm{TiO}_{2}(\%)$ & - & 1,49 & \multirow{2}{*}{$\begin{array}{l}\text { Resistência à compressão do } \\
\text { cimento }-3^{\circ} \text { dia } \\
\end{array}$} & \multirow[b]{2}{*}{30,70} & \multirow[t]{2}{*}{-} \\
\hline $\mathrm{SO}_{3}(\%)$ & 2,72 & - & & & \\
\hline $\mathrm{CO}_{2}(\%)$ & 2,08 & - & \multirow{2}{*}{$\begin{array}{l}\text { Resistência à compressão do } \\
\text { cimento }-7^{\circ} \text { dia }\end{array}$} & \multirow[b]{2}{*}{34,00} & \multirow[t]{2}{*}{ - } \\
\hline CaO livre & 1,48 & - & & & \\
\hline $\mathrm{CaO}$ total & 64,32 & 0,02 & Atividade Pozolânica (\%) & & 94,15 \\
\hline
\end{tabular}

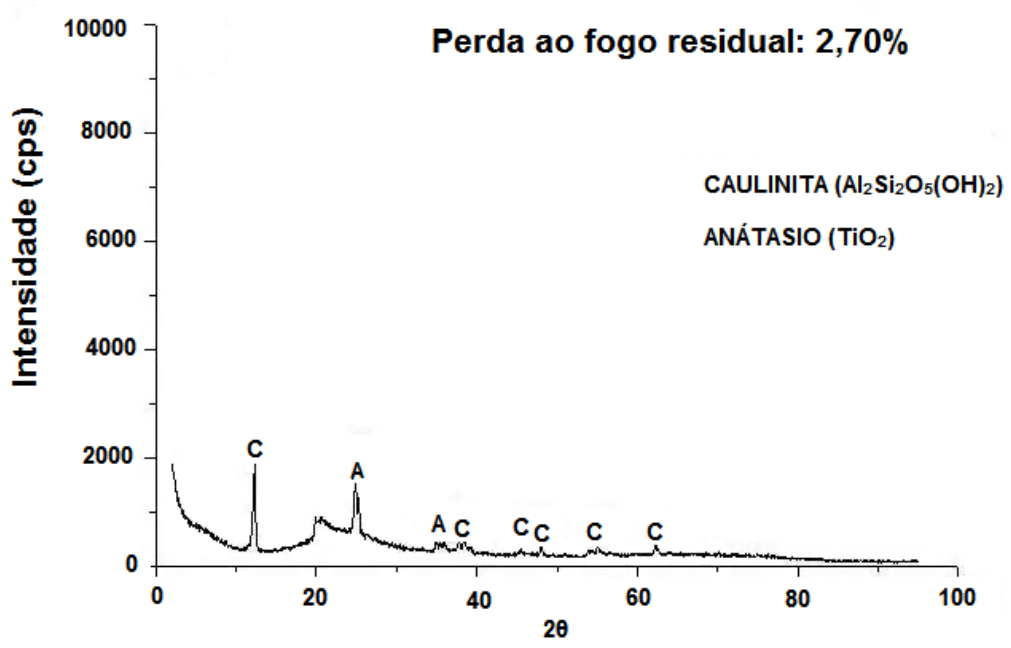

Figura 1: Difração de raios-X da pozolana, após o processo de calcinação.

Utilizou-se o superplastificante à base de naftaleno sulfonado, constituído de 37 a $39 \%$ de sólidos. Na determinação da quantidade final de água a ser adicionado à mistura, adotou-se o valor de 38\% de sólidos para corrigir a relação a/(c+MCAR).

\subsection{Programa Experimental}

Para a verificação da influência deste material, fez-se necessário a fixação das variáveis controláveis da pesquisa. Adotou-se 5 níveis para cada variável controlável, conforme segue: teor de substituição de MCAR: 0\%, 5\%, 10\%, 15\% e 20\%; relação a/(c+MCAR): 0.25, 0.28, 0.35, 0.46 e 0.60; idade: 1,7,14,28 e 91 dias.

Em função do número de variáveis existentes, adotou-se um projeto estatístico, que teve como método o fracionamento.

O método de fracionamento é baseado em observações (nesta pesquisa estas observações são os ensaios) realizadas em pontos específicos do espaço amostral de maneira que o comportamento das variáveis de resposta é mantido, obtendo-se as informações relevantes com um menor número de corpos de prova. Este projeto estatístico é explicado por [7], sendo definido como projeto fatorial fracionado somado a pontos axiais e centrais que proporcionam o estudo dos efeitos produzidos nas variáveis de resposta, pela mudança de nível dos fatores controláveis. Um exemplo gráfico deste projeto é colocado na figura 2, onde observa-se que os pontos formam uma estrela e em cubo, sendo que os pontos que formam a estrela possibilitam informações das interações entre os fatores e os pontos que formam o cubo identificam os efeitos de mais alta ordem. 


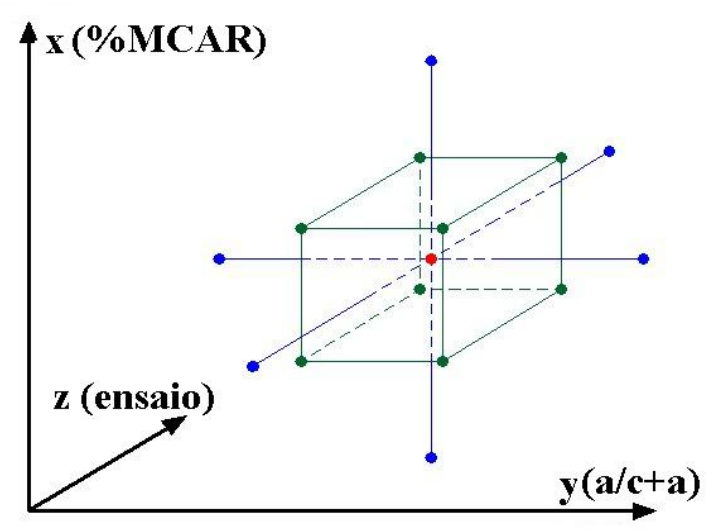

Figura 2: A estrela e o cubo do projeto estatístico fatorial fracionado.

Optou-se por utilizar dois corpos-de-prova de diferentes betonadas para cada combinação. Mais detalhes sobre o programa experimental foram publicados por [8]. Adotou-se o método de dosagem proposto por [9]. Para o controle da trabalhabilidade, utilizou-se o valor de $120 \pm 20 \mathrm{~mm}$ para a realização do ensaio de abatimento do tronco de cone, de acordo com [10], sendo este procedimento o referencial para a definição do teor de aditivo a ser utilizado, desta forma o teor de aditivo empregado variou entre as misturas realizadas, ficando contudo dentro do especificado pelo fabricante. A tabela 3 apresenta as quantidades de materiais, onde ressalta-se que a quantidade de água final inclui a água inserida no aditivo. É Interessante ressaltar que a relação a/(C+MCAR) adotada não levou em conta a quantidade de água contida no aditivo.

Tabela 3: Proporcionamento dos materiais para a produção dos concretos

\begin{tabular}{|c|c|c|c|c|c|c|}
\hline $\begin{array}{l}\text { RELAÇÃOO } \\
\text { A/(C+MCAR) }\end{array}$ & \begin{tabular}{|l|} 
MCAR \\
$\%$
\end{tabular} & \begin{tabular}{|l|} 
ÁGUA \\
$\left(K G / M^{3}\right)$
\end{tabular} & $\begin{array}{l}\text { CIMENTO } \\
\left(K^{\prime} / M^{3}\right)\end{array}$ & \begin{tabular}{|l|} 
MCAR \\
$\left(K G / M^{3}\right)$
\end{tabular} & $\begin{array}{l}\text { AGREGADO } \\
\text { MIÚDO } \\
\text { (KG/M }^{3} \text { ) } \\
\end{array}$ & \begin{tabular}{|l|} 
AGREGADO \\
GRAÚDO \\
(KG/M $\left.{ }^{3}\right)$ \\
\end{tabular} \\
\hline \multirow[t]{3}{*}{0,25} & 0 & 144 & 575 & - & 654 & 1085 \\
\hline & 10 & 143 & 517 & 46 & 654 & 1085 \\
\hline & 20 & 140 & 460 & 93 & 654 & 1085 \\
\hline \multirow[t]{3}{*}{0,28} & 5 & 153 & 520 & 22 & 669 & 1069 \\
\hline & 10 & 152 & 492 & 44 & 669 & 1069 \\
\hline & 15 & 151 & 465 & 66 & 669 & 1069 \\
\hline \multirow[t]{3}{*}{0,35} & 0 & 171 & 491 & - & 700 & 1038 \\
\hline & 10 & 171 & 442 & 40 & 700 & 1038 \\
\hline & 20 & 167 & 393 & 79 & 700 & 1038 \\
\hline \multirow[t]{3}{*}{0,46} & 5 & 193 & 399 & 17 & 739 & 997 \\
\hline & 10 & 191 & 378 & 34 & 739 & 997 \\
\hline & 15 & 189 & 357 & 51 & 739 & 997 \\
\hline \multirow[t]{3}{*}{0,60} & 0 & 214 & 358 & - & 773 & 962 \\
\hline & 10 & 210 & 323 & 29 & 773 & 962 \\
\hline & 20 & 208 & 287 & 58 & 773 & 962 \\
\hline
\end{tabular}

$\mathrm{Na}$ execução dos traços utilizou-se uma betoneira de eixo vertical, realizando-se a seguinte sequencia de colocação dos materiais: agregado graúdo; metade do agregado miúdo; uma parte da água; cimento; MCAR; metade do agregado miúdo; restante da água. A moldagem e o processo de cura seguiram os procedimentos de [11]. Com 24 horas de cura em ambiente de laboratório, foi feita a desforma e a colocação dos corpos-de-prova em câmara úmida até o momento do ensaio, realizado segundo [12]. 


\section{ANÁLISES DOS RESULTADOS E DISCUSSÕES}

Os resultados do ensaio de módulo de elasticidade foram obtidos com o LVDT (transformador diferencial variável linear). O modelo matemático desta propriedade está colocado na equação 1 e apresenta um valor de $\mathrm{r}^{2}$ de $88,70 \%$. O valor é estatisticamente consistente, pois demonstra que aproximadamente $89 \%$ da variabilidade dos resultados é decorrente do efeito isolado das variáveis e da interação entre as mesmas, fazendo com que os valores obtidos neste modelo ficassem muito próximos aos obtidos em ensaio. O emprego desta equação possibilitou a montagem dos gráficos empregados nesta análise. Contudo, para a obtenção desses valores, através da equação 1, fez-se necessário que as variáveis controladas (relação água/(c + MCAR), teor de substituição e idade de rompimento) deste experimento fossem codificados para níveis entre -1 e 1, para que as constantes das equações a serem obtidas apresentassem a mesma ordem de grandeza. No entanto, face à possibilidade de se ter valores iguais a zero no denominador, ajustou-se os níveis codificados de cada um dos parâmetros empregados, para valores entre 0,5 e 1,5. Na tabela 4 são colocados os níveis reais e codificados de cada variável.

$$
\begin{aligned}
\text { Ec }= & 14,1332+0,00163592 /\left(\text { Teor }^{4} * \text { Idade }^{8}\right)-0,00496357 * \text { AC }^{3} / \text { Idade }^{9}+17,3975 * \text { Teor }^{0,2} \\
& +9,76265 / \text { AC }^{0,5}-1,83621 * \text { Teor }^{0,01 *} \mathrm{AC}^{3}-0,0496533 / \text { Idade }^{8}
\end{aligned}
$$

\begin{tabular}{|c|c|c|c|c|c|c|}
\hline RELAÇÃO Al(C+MCAR) - AC & $\begin{array}{l}\text { NÍVEL REAL } \\
\text { NÍVEL CODIFICADO }\end{array}$ & $\begin{array}{l}0,25 \\
0,50 \\
\end{array}$ & $\begin{array}{l}0,28 \\
0,585\end{array}$ & $\begin{array}{l}0,35 \\
0,785 \\
\end{array}$ & $\begin{array}{l}0,46 \\
1,10 \\
\end{array}$ & $\begin{array}{l}0,60 \\
1,50 \\
\end{array}$ \\
\hline IDADE (DIAS) & $\begin{array}{l}\text { NÍVEL REAL } \\
\text { NÍVEL CODIFICADO }\end{array}$ & $\begin{array}{l}1 \\
0,50 \\
\end{array}$ & $\begin{array}{l}7 \\
0,565 \\
\end{array}$ & $\begin{array}{l}14 \\
0,645\end{array}$ & $\begin{array}{l}28 \\
0,80 \\
\end{array}$ & $\begin{array}{l}91 \\
1,50\end{array}$ \\
\hline TEOR DE SUBSTITUIÇÃO (\%) & $\begin{array}{l}\text { NÍVEL REAL } \\
\text { NÍVEL CODIFICADO }\end{array}$ & \begin{tabular}{|l}
0 \\
0,50 \\
\end{tabular} & \begin{tabular}{|l}
5 \\
0,75 \\
\end{tabular} & $\begin{array}{l}10 \\
1,00 \\
\end{array}$ & $\begin{array}{l}15 \\
1,25 \\
\end{array}$ & $\begin{array}{l}20 \\
1,50 \\
\end{array}$ \\
\hline
\end{tabular}

Tabela 4: Níveis reais e codificados das variáveis independentes utilizadas

Os parâmetros da equação apresentaram valores-p em patamares inferiores a 0,05, demonstrando que o modelo proposto é estatisticamente significativo para um nível de confiança de $95 \%$, não sendo aconselhável suprimir nenhuma das variáveis empregadas. Na análise de variância do modelo, teve-se um alto valor de F, em relação ao valor de F calculado, o que rejeita a hipótese nula, de que não existe relação entre as variáveis independentes e a resposta considerada. Nesta análise o valor-p foi zero, indicando que há uma relação estatisticamente significativa entre as variáveis independentes e a resposta considerada.

\subsection{Efeito dos fatores a/(c+MCAR) e idade (grau de hidratação) no módulo de elasticidade do concreto.}

Na figura 3 tem-se os gráficos de tendência do comportamento do efeito isolado da relação a/(c + MCAR) e da idade de rompimento, respectivamente, no módulo de elasticidade. Os gráficos foram obtidos variando os valores codificados do efeito focalizado e mantendo-se as demais variáveis no ponto médio do intervalo codificado de cada uma delas.

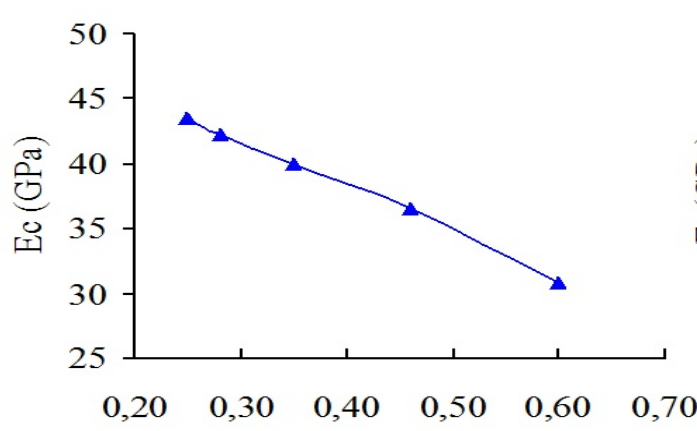

Relação água/(c+MCAR)

(a)

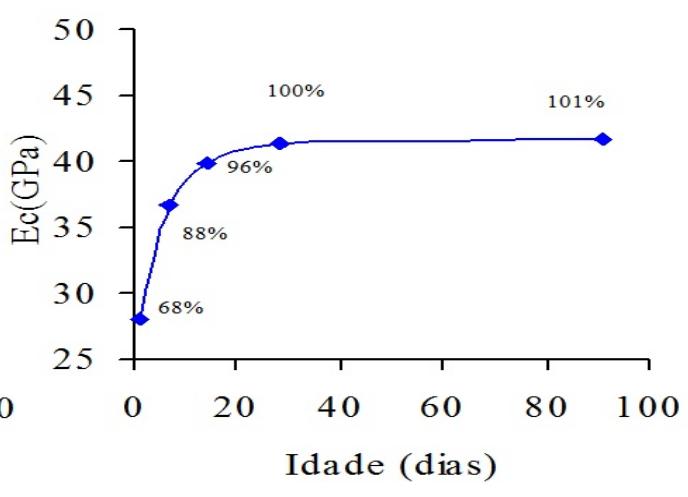

(b)

Figura 3: Módulo de elasticidade em função do efeito isolado da: a) relação a/(c+MCAR) b) Idade de rompimento. 
Como era esperado, o gráfico (a) do efeito isolado da relação a/(c+MCAR) da figura 3 apresenta uma diminuição dos valores do módulo de elasticidade conforme aumenta-se os valores da relação. Nas maiores relações a/(c+MCAR), tem-se um maior afastamento dos grãos de cimento fazendo com que haja uma maior presença, principalmente na zona de transição, dos cristais de hidróxido de cálcio e de etringita, resultando num enfraquecimento das ligações no concreto endurecido.

Com relação ao gráfico (b) referente ao efeito isolado da idade de rompimento, contido na figura 3 , tem-se novamente que os resultados encontraram-se dentro do esperado, ou seja, aumentaram com o aumento da idade de rompimento. Neste gráfico, verifica-se que a taxa de evolução do módulo de elasticidade é acentuada, chegando-se a obter no $1^{\circ}$ dia $68 \%$ do valor atingido ao $28^{\circ}$ dia. Novamente, é interessante ressaltar que o comportamento apresentado nos gráficos colocados na figura 3 tendem a variar em decorrência de outras variáveis, como o teor de substituição de MCAR.

Na verificação da interação entre a relação a/(c+MCAR) e a idade de rompimento, apresentada na figura 4, foi necessária a manutenção da outra variável (teor de substituição) no ponto médio.

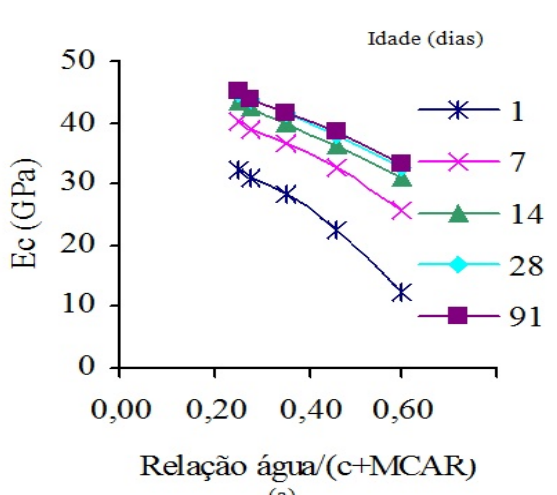

(a)

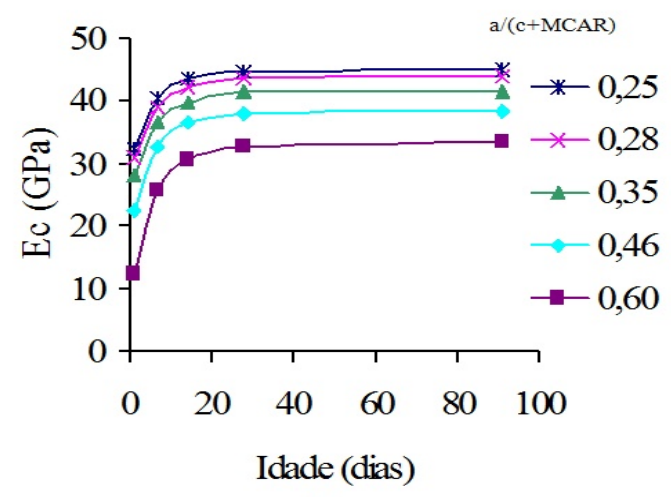

(b)

Figura 4: Módulo de elasticidade em função da interação relação a/(c+MCAR) x idade de rompimento: a) gráfico Ec (relação a/(c+MCAR)) b) gráfico Ec (idade de rompimento).

Com relação a esta interação, tem-se, segundo os gráficos colocados na figura 4, um crescimento mais lento do módulo de elasticidade nas primeiras idades quando adota-se maiores valores de relação a/(c+MCAR). Por outro lado, nas baixas relações a/(c+MCAR), o crescimento dos valores de módulo de elasticidade tende a ser maior nas primeiras idades. Nas duas situações tem-se um crescimento muito reduzido após o $28^{\circ}$ dia.

\subsection{Efeito da adição de metacaulim de alta reatividade no módulo de elasticidade do concreto}

Para a obtenção do efeito isolado do teor de substituição de MCAR no módulo de elasticidade, colocada na figura 5, fixou-se a idade de rompimento no $28^{\circ}$ dia, para que se pudesse obter a contribuição média, dentre as relações água/(c+MCAR), no $28^{\circ}$ dia de idade dos concretos, para todos os teores de substituição estudados. 


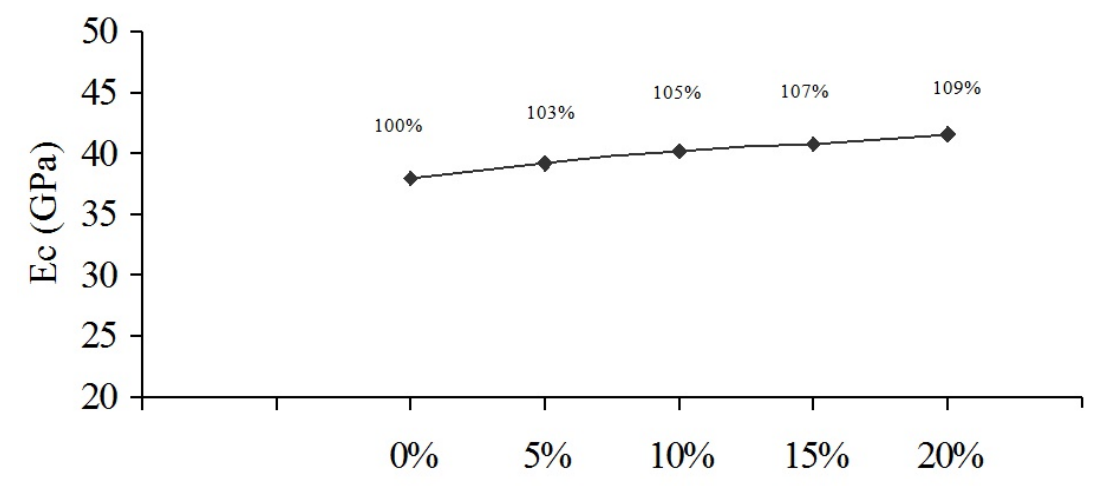

Teor de substituição

Figura 5: Módulo de elasticidade em função do efeito isolado do teor de substituição.

De acordo com os valores colocados na figura 5, tem-se uma melhoria desta propriedade com o aumento do teor de substituição de MCAR no concreto. A contribuição decorrente do uso do MCAR é semelhante à obtida por [13,14], e inferior aos valores colocados por [4]. Estas diferenças podem ser creditadas às variações das características físicas e químicas do MCAR. Interessante ressaltar que os trabalhos realizados pelos referidos autores envolveram o MCAR proveniente de argila caulinitica e não o proveniente de rejeito industrial, que o caso do presente trabalho. Não se encontrou na bibliografia existente trabalhos com este tipo de material em estudos envolvendo o modulo de elasticidade.

Na comparação das melhorias obtidas com o MCAR com as de outras pozolanas de alta reatividade, constata-se um desempenho diferenciado dos resultados obtidos neste trabalho. No caso da sílica ativa, [15] também constatou a significância desta variável, onde obteve ao $28^{\circ}$ dia, com um teor de adição de $10 \%$, uma melhoria média, dentre as várias relações a/(c+sa) utilizadas, de aproximadamente $4 \%$ em relação ao concreto de referência, um pouco inferior à melhoria obtida no presente trabalho, mesmo tendo-se empregado o MCAR por meio de substituição.

Quanto a cinza de casca de arroz, constata-se, tendo com base os modelos matemáticos relatados por [16], que no $28^{\circ}$ dia há uma diminuição dos valores do módulo de elasticidade, sendo que esta diminuição aumenta com o aumento do teor de substituição. Após esta idade este comportamento tende-se a alterar, chegando-se a obter, no $91^{\circ}$ dia, um aumento dos valores, em relação ao concreto de referência, com o aumento do teor de substituição. Apesar deste aumento para as maiores idades, a contribuição média obtida pela pesquisadora ainda foi menor que a obtida neste trabalho com o uso do MCAR, no $91^{\circ}$ dia.

A diferença de comportamento obtido entre as pozolanas de alta reatividade pode ser decorrente da finura da sílica ativa em relação ao MCAR e à cinza de casca de arroz. Esta situação faz com que as partículas do MCAR e da cinza de casca de arroz, proporcionem a atuação destes materiais não só como fíler, mas também como micro-agregados nas misturas. No caso do MCAR, o melhor desempenho também pode ter como causa um maior efeito nesta propriedade, dos produtos resultantes da reação pozolânica deste material, como por exemplo a gelenita.

\subsection{Efeito da adição de metacaulim de alta reatividade em função da relação a/(c+MCAR), no módulo de elasticidade do concreto}

Na figura 6 pode ser visualizado o efeito desta interação sobre o módulo de elasticidade. Os valores para a montagem dos gráficos foram obtidos a partir variação dos valores codificados da relação a/(c+MCAR) para cada valor codificado do teor de substituição, mantendo-se a outra variável (idade) no ponto médio. 


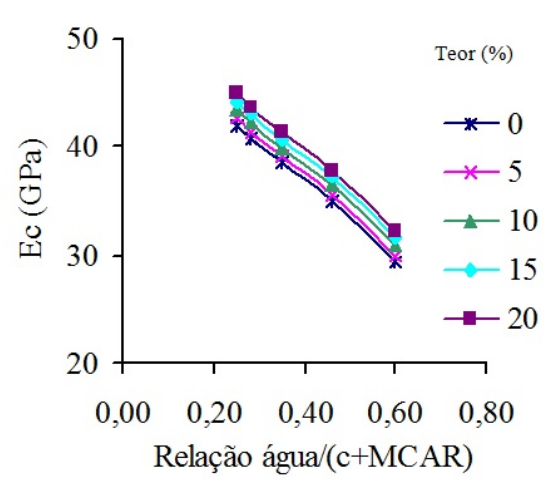

(a)

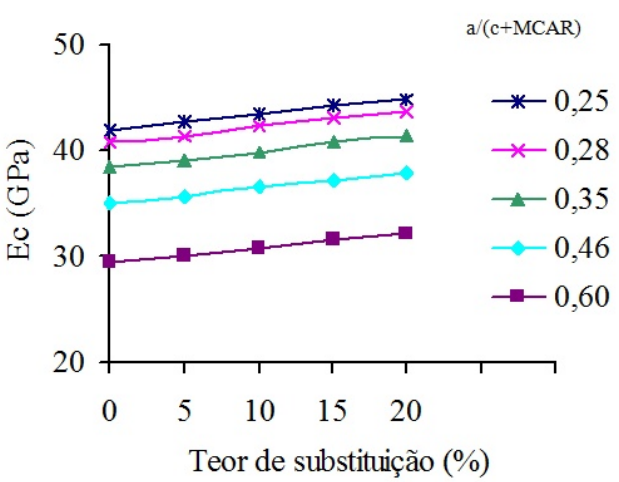

(b)

Figura 6: Módulo de elasticidade em função da interação teor de substituição x relação a/(c+MCAR): a) gráfico Ec (relação a/(c+MCAR)) b) gráfico Ec (teor de substituição).

Segundo a figura 6, constata-se uma melhor eficiência do MCAR quando do uso de maiores relações $\mathrm{a} /(\mathrm{c}+\mathrm{MCAR})$. Este comportamento pode ser justificado pelo fato de que nas maiores relações a/(c+MCAR), tem-se uma maior porosidade, que ocasiona uma maior eficiência do efeito pozolânico pelo preenchimento dos vazios da mistura, bem como pelo efeito filer. A comparação dos resultados com os resultados de outros trabalhos que focalizaram o uso do MCAR fica dificultada pela inexistência de trabalhos que abordem esta interação em relação ao módulo de elasticidade.

A colocação da idade no ponto médio fez com que os valores colocados na figura 6 sejam referentes ao $14^{\circ}$ dia. Nas menores ou maiores idades este comportamento pode ser diferente, conforme constatado na interação teor de substituição de MCAR x idade de rompimento, colocada a seguir.

\subsection{Efeito da adição de metacaulim de alta reatividade em função da idade, no módulo de elasticidade do concreto}

Os gráficos da figura 7 representam o comportamento do módulo de elasticidade em função desta interação e foram obtidos variando os valores codificados do teor de substituição para cada valor codificado da idade, mantendo-se a outra variável (relação a/(c+MCAR)) no ponto médio.
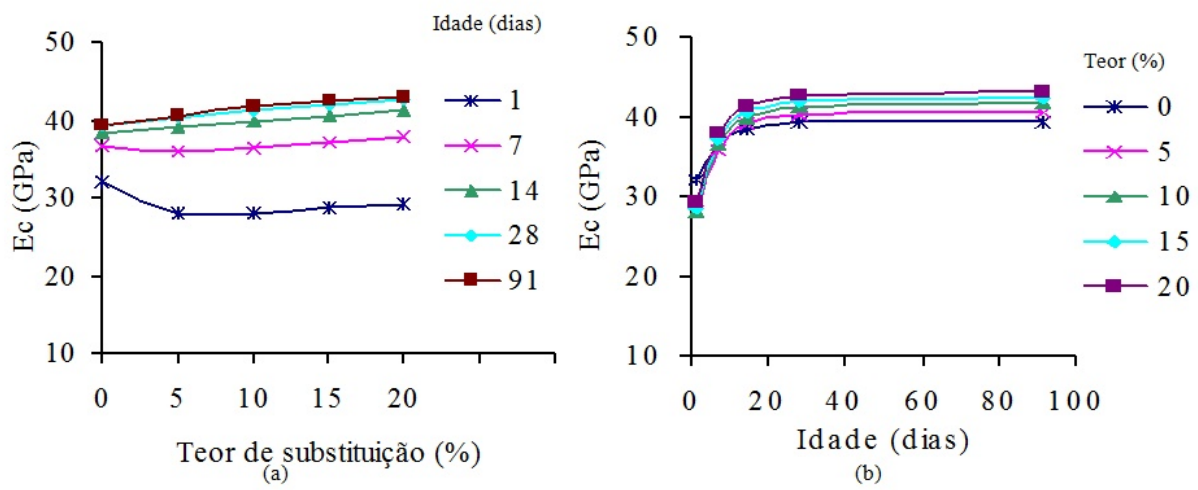

Figura 7: Módulo de elasticidade em função da interação teor de substituição x idade de rompimento: a) gráfico Ec (teor de substituição) b) gráfico Ec (idade).

Constata-se na figura 7 que o aumento do teor de substituição provocou a redução dos valores de módulo apenas nas primeiras idades. Após o $14^{\circ}$ quarto dia, o crescimento dos valores permaneceu praticamente inalterada, independente do teor de substituição adotado. Assim sendo, teve-se nas misturas com MCAR um crescimento médio dos valores de módulo, em relação ao $28^{\circ}$ dia, de $69 \%$ e $89 \%$, para as idades de 1 e 7 dias, enquanto que nas misturas sem MCAR o crescimento foi de $82 \%$ e $93 \%$ para as referidas idades. Nas demais 
idades, o crescimento médio foi de $97 \%$ e $101 \%$ para as idades de 14 e 91 dias, respectivamente, independente do teor de substituição. Este comportamento é semelhante ao obtido por [4,13].

Em relação ao concreto de referência, observa-se que nas primeiras idades a substituição de cimento por MCAR tende a provocar uma diminuição dos valores de módulo. Esta diminuição tende a ser novamente resultado da insuficiência do efeito microfiler em compensar a retirada de cimento, bem como a maior lentidão da reação pozolância do MCAR. No $14^{\circ}$ dia, já se observa um crescimento dos valores de módulo com o aumento do teor de substituição, onde se verificou melhorias médias de 5\%, 6\% e 7\% para as idades de 14 , 28 e 91 dias, respectivamente. [13,14] obtiveram melhorias semelhantes, principalmente para a idade de 28 dias, com o uso do MCAR. Outro trabalho realizado por [4] também constatou a melhoria nesta propriedade, contudo, observa-se que as melhorias em relação ao concreto de referência foram de $35 \%$, 36\% e 27\%, para as idades de 7, 28 e 90 dias, respectivamente, ou seja, maiores que os obtidos nesta pesquisa. Esta variação pode ser creditada às diferenças existente no MCAR usado nos trabalhos referenciados, tais como finura $\mathrm{e}$ teores de $\mathrm{Al}_{2} \mathrm{O}_{3}$, que exercem grande influência nos efeitos filer e pozolânico, respectivamente, bem como o tipo de cimento adotado.

Com a colocação da relação a/(c+MCAR) no ponto médio, os valores colocados na figura 7 foram referentes à relação de 0,35 . Para as menores ou maiores relações a/(c+MCAR) este comportamento pode ser diferente.

De um modo geral, tem-se no módulo de elasticidade, pelos resultados obtidos que o teor ótimo de substituição possa ser superior ao máximo valor colocado neste trabalho, sendo desta forma superior ao teor ótimo colocado para a sílica ativa, onde segundo [17], este deve ser inferior a 16\%, quando se focaliza a melhoria no módulo de elasticidade.

\section{CONCLUSÕES}

Assim sendo, são colocadas a seguir as conclusões obtidas com a realização deste trabalho:

1. Os valores do módulo de elasticidade elevaram-se ao aumentar-se a idade, e diminuíram com o aumento da relação água/(c+MCAR), como era esperado.

2. Na interação entre a relação a/(c+MCAR) e a idade (grau de hidratação) teve-se um comportamento diferenciado do módulo de elasticidade, para as diversas relações a/(c+MCAR). Nas menores relações a/(c+MCAR) o crescimento foi maior nas primeiras idades, enquanto que nas maiores relações a/(c+MCAR) o crescimento foi mais lento. Nas duas situações, teve-se pouco crescimento entre o $28^{\circ}$ e o $91^{\circ}$ dia.

3. Na verificação do efeito isolado do teor de substituição de cimento por MCAR, teve-se uma melhoria diretamente proporcional ao teor de substituição adotado, chegando-se a obter uma melhoria média de $9 \%$, dentre as várias relações a/(c+MCAR) utilizadas, no $28^{\circ}$ dia de idade com um teor de substituição de $20 \%$.

4. A interação entre a relação a/(c+MCAR) e o teor de substituição também mostrou-se significativo. Nesta interação, verificou-se que nas menores relações a/(c+MCAR) a melhoria no módulo de elasticidade decorrente do aumento do teor de substituição foi inferior à obtida nas maiores relações a/(c+MCAR).

5. Na interação da idade com o teor de substituição, teve-se um crescimento diferenciado dos valores de módulo de elasticidade, em relação às idades estudadas. No $1^{\circ}$ e no $7^{\circ}$ dia, verificou-se uma diminuição dos valores com o aumento do teor de substituição, enquanto que nas demais idades, verifica-se um aumento dos valores com o aumento do teor de substituição de MCAR.

De uma forma geral, pode-se concluir, pelo modelo obtido, que o MCAR tende a resultar em melhorias nesta propriedade. Esta melhoria, além de resultar em vantagens técnicas, ainda proporciona uma excelente opção de utilização de um resíduo que provoca grande agressão ambiental. Contudo, mesmo com os resultados obtidos, deve-se ressaltar a necessidade de pesquisas adicionais visando consolidar o conhecimento obtido no presente trabalho.

\section{AGRADECIMENTOS}

Os autores agradecem o suporte financeiro fornecido pelo CNPq (Conselho Nacional de Desenvolvimento Científico e Tecnológico) para a realização deste trabalho. 


\section{BIBLIOGRAFIA}

[1] BARATA, M. S., Concreto de alto desempenho no estado do Pará: Estudo de viabilidade técnica e econômica de produção do CAD com materiais disponíveis em Belém, através do emprego de adição de sílica ativa e metacaulim, Tese de D.Sc., PPGEC da UFRGS, Porto Alegre, RS, Brasil, 1998.

[2] TAYLOR, P.C, BURG, R.G, “Low-cost, durable concrete”, Concrete Construction, pp.33-34, Apr. 1999.

[3] BALOGH, A, “High-reactivity metakaolin”, Concrete construction,v.40,n.7, p.9, July. 1995.

[4]CALDARONE, M.A., GRUBER, K. A., BURG, R.G., "High-reactivity Metakaolin: A New Generation Mineral Admixture”, Concrete International, v.16, n.11, pp. 37-40, Nov. 1994.

[5] BARATA, M. S, Aproveitamento dos resíduos cauliníticos das indústrias de beneficiamento de caulim da região amazônica como matéria prima para fabricação de um material de construção (pozolanas), Tese de D.Sc, PPGEC da UFPA, Belém, PA, Brasil, 2007.

[6] FLORES, S.M.P., Aproveitamento do rejeito de caulim na produção de alumina para cerâmica e sílica ativa de baixa granulometria, Tese de D.Sc., Centro de Ciências Naturais da UFPA, Belém, PA, Brasil, 1998.

[7] MONTGOMERY, D.C. Design and analysis of experiments, New York, Jonh Willey \& Sons, 1986.

[8] SOUZA, P.S.L., Verificação da influência do uso do metacaulim de alta reatividade na propriedades mecânicas do concreto de alta resistência, Tese de D.Sc., PPGEC da UFRGS, Porto Alegre, RS, Brasil, 2003.

[9] METHA, P.K., AÏTCIN, P.C., "Principles underlying production of high-performance concrete”, Cement, Concrete and Aggregates, v.12, n.2, pp.70-78, Winter.1990.

[10] ASSOCIAÇÃO BRASILEIRA DE NORMAS TÉCNICAS. Concreto - Determinação da consistência pelo abatimento do tronco de cone: NBR 7223. Rio de Janeiro, 1992.

[11] ASSOCIAÇÃO BRASILEIRA DE NORMAS TÉCNICAS. Confecção e cura de corpos-de-prova de concreto cilíndricos ou prismáticos: NBR 5738. Rio de Janeiro, 1984.

[12] ASSOCIAÇÃO BRASILEIRA DE NORMAS TÉCNICAS. Concreto - Determinação do módulo de deformação estática e diagrama - Tensão-Deformação: NBR 8522. Rio de Janeiro, 1984.

[13] QUIAN, X., LI, Z., “The relationships between stress and strain for high-performance with metakaolin”, Cement and Concrete Research, v. 31, n. 11, pp1607-1611, 2001.

[14] ZHANG, M.H., MALHOTRA, V.M., "Characteristics of thermally activated alumino-silicate pozzolanic material and its use in concrete”, Cement and Concrete Research, v. 25, n. 8, pp1713-1725, Jul. 1995.

[15] DAL MOLIN, D.C.C., Contribuição ao estudo das propriedades mecânicas dos concretos de alta resistência com e sem adição de microssílica, Tese de D.Sc., Escola Politécnica da USP, São Paulo, SP, Brasil, 1995.

[16] SENSALE, G.R.B de., Estudo comparativo entre as propriedades mecânicas dos concretos de alta resistência com cinza de casca de arroz, Tese de D.Sc., PPGEC da UFRGS, Porto Alegre, RS, Brasil, 2000.

[17] SABIR, B.B., "High-strength condensed silica fume concrete”, Magazine of Concrete Research,v.47, n. 172, pp219-226, Sep. 1995. 